\title{
Artificial intelligence allows quick and easy diagnosis of pancreatic cancer
}

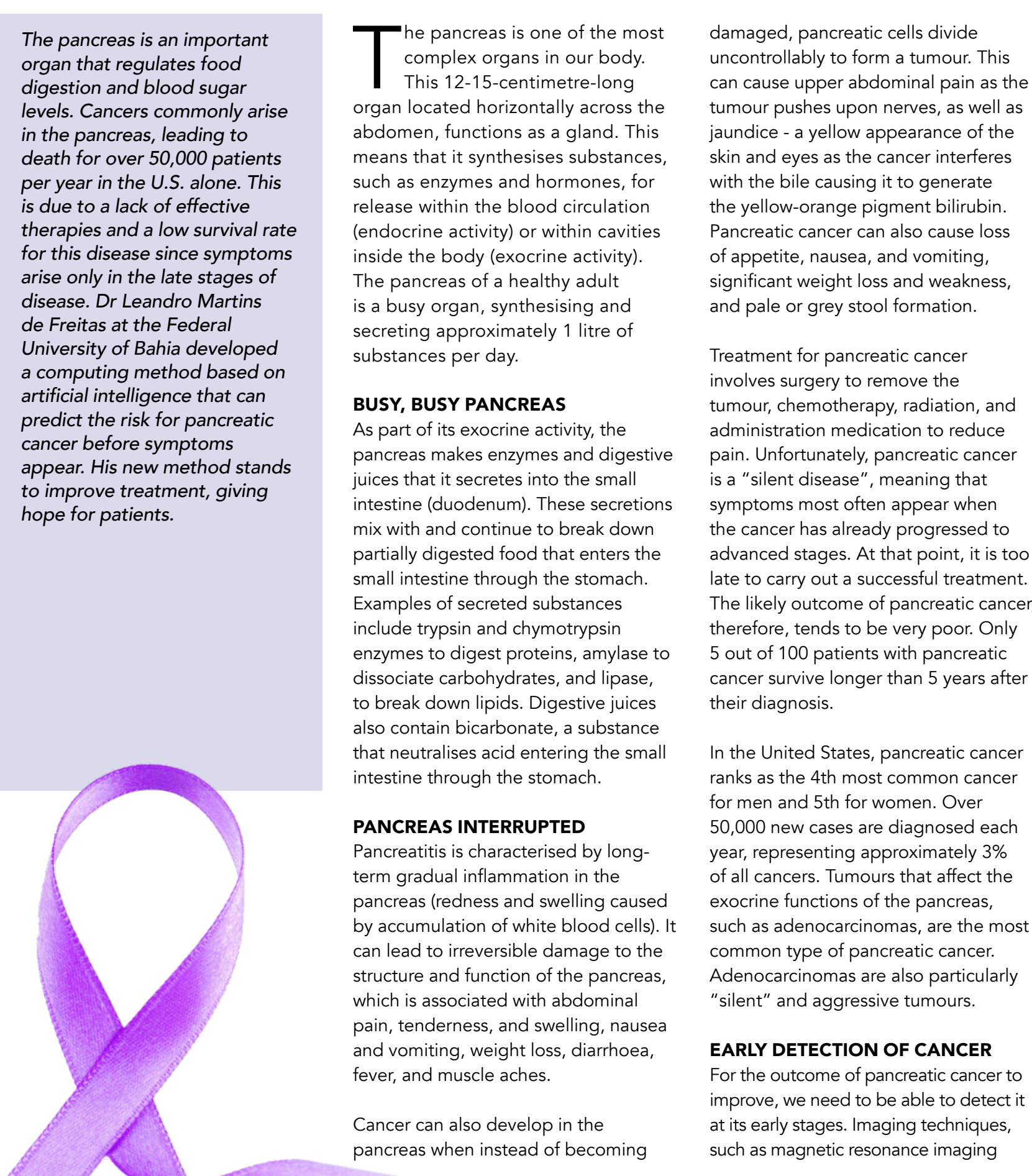

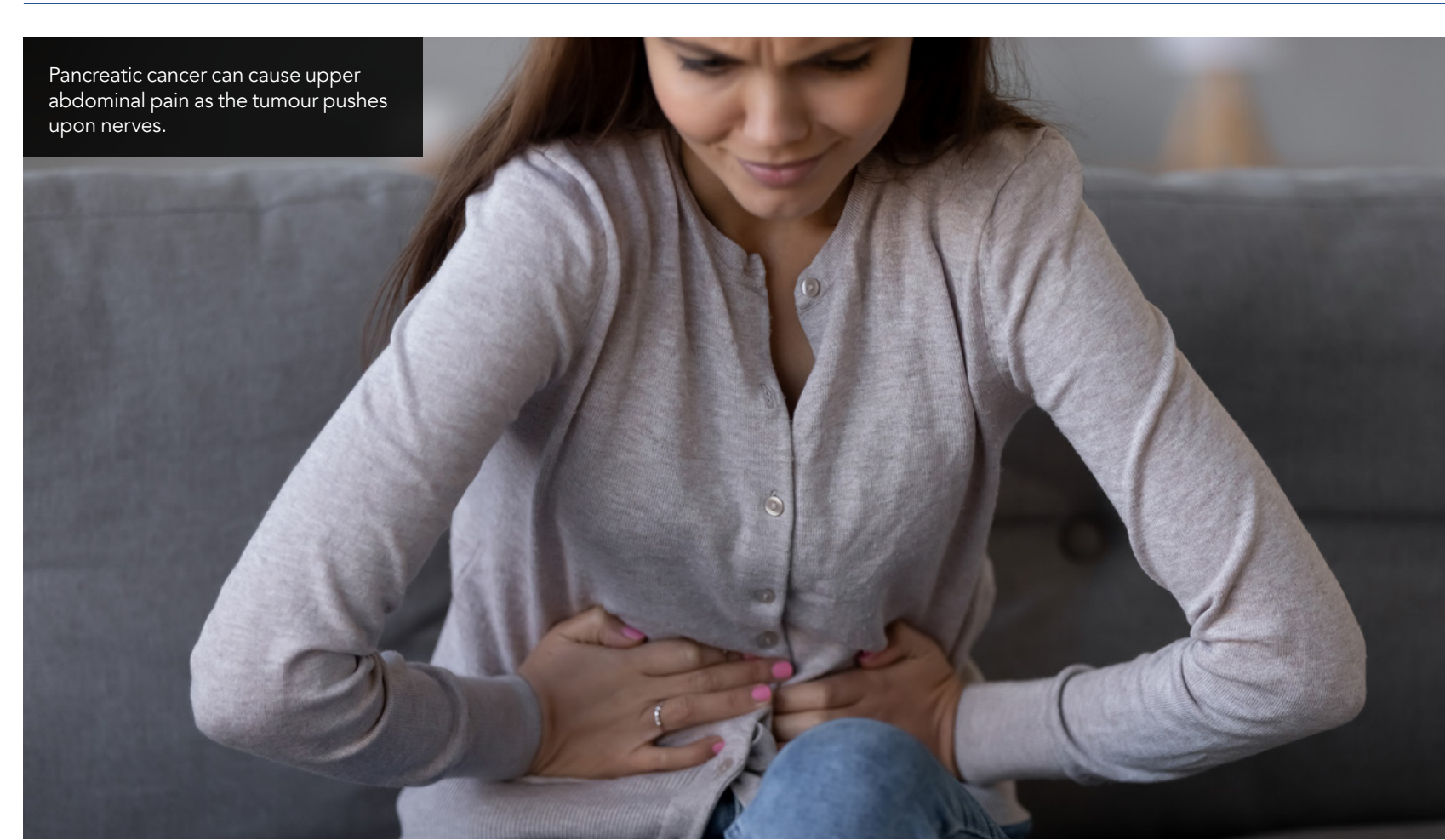

(MRl) and computed tomography (CT) scans, may accurately diagnose the cancer, but are expensive and uncomfortable for patients. For years, scientists have tried to achieve easy, early, accurate, and cheap diagnosis by studying the levels of all genes (transcriptome) and proteins (proteome) that are present in our bodies when cancer appears. This type of study is referred to as "biomarker" analysis. It offers the possibility of improving the detection of cancer, and therefore treat it more effectively. One biomarker th has been used in the clinic is CA 19-9, a sugar-bound molecule whose level in patients. However its pevels often do not rise early enough to signal an alarm for doctors and patients.

Dr Leandro Martins de Freitas at the Federal University

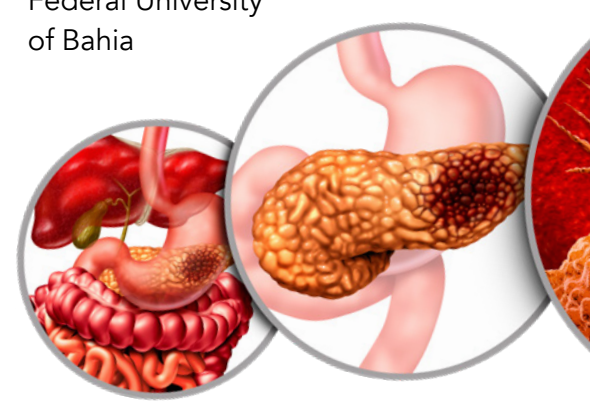

Cancer can develop in the pancreas when
pancreatic cells divide uncontrollably to pancreatic cells
form a tumour.
Symptoms of pancreatic cancer often appear when it is too late for therapy. (UFBA), Brazil, has recently published a new study that identified five new biomarkers for pancreatic published in 10 independent Dr Freitas and his team harnessed as gene expression levels) in 463 the power of publicly available information (i.e., they performed a "meta-analysis) regarding the levels of genes and proteins present in patients with adenocarcinoma. Findings were ion 187 normal samples. They found that the expression levels of 40 genes were different between the two groups; 39 genes had higher levels in
adenocarcinoma patients, and one had

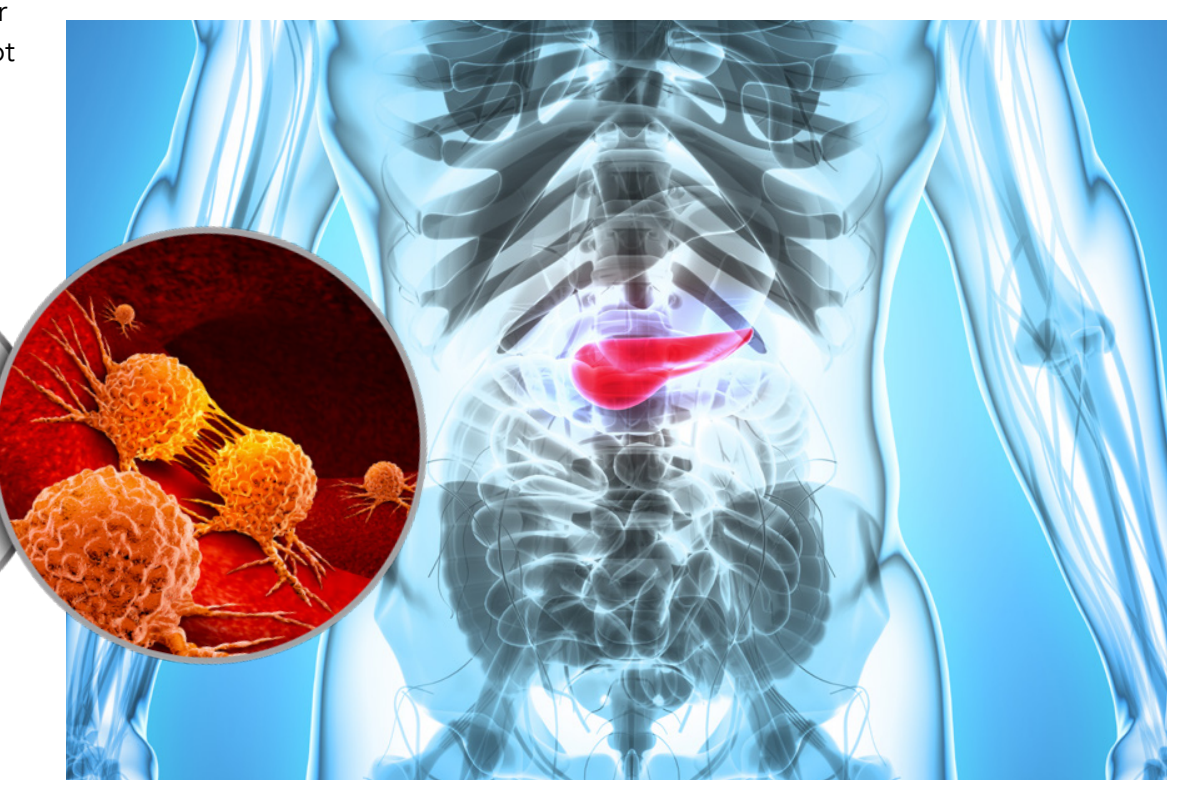




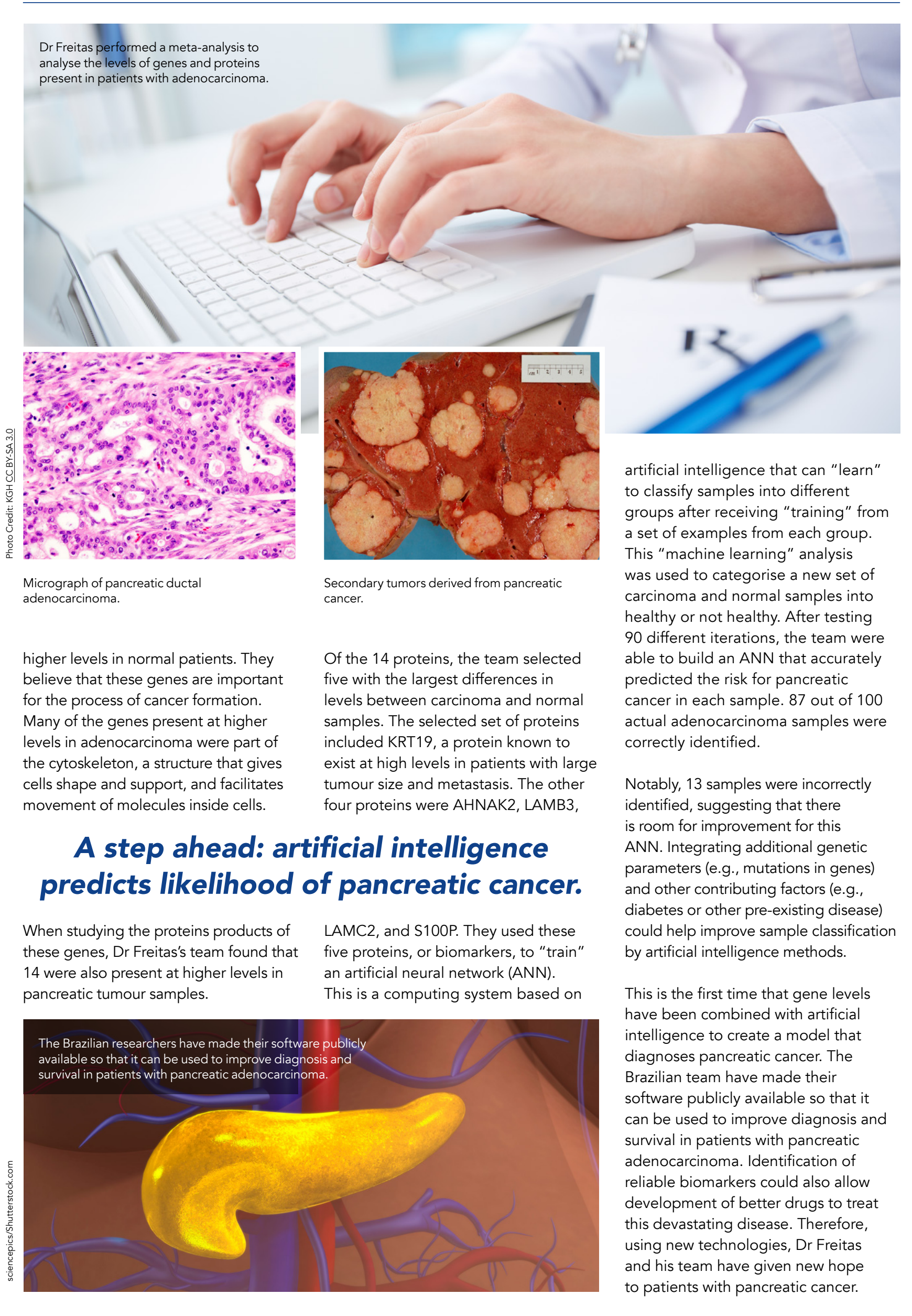

\section{-1. Behind the Research Dr Leandro Martins de Freitas}

E: leandromartins@ufba.br： T: +55 (77) 99170-4669 W: http://www.genebio.ufba.br/freitas/

\section{Research Objectives}

Leandro Martins de Freitas explores the use of genetics and gene expression to assist cancer diagnosis.

\section{Detail}

Bio

Leandro Martins de Freitas completed his B.Sc. in Biological Science in 2004, and his M.Sc in Genetics Federal University of Minas Gerais (UFMG) in 2011. Since 2009, Dr de Freitas has taught Genetics, Bioinformatics and Evolution at Federal University of Bahia (UFBA). He has professional experience in sequence and expression analysis, epitope and immunological prediction.

Funding

CAPES - Coordenação de Aperfeiçoamento de Pessoal de Nível Superior

Collaborator Palloma Porto Almeida

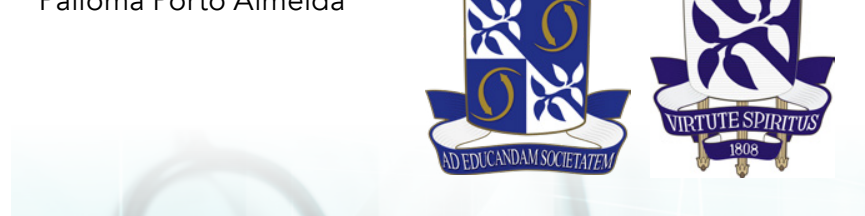

\section{References}

Almeida, PP., Cardoso, CP., Freitas, LM. PDAC-ANN: an artificial neural network to predict Pancreatic Ductal Adenocarcinoma based on gene expression. BMC Cancer. 2020;20(1):82. doi:10.1186/s12885-020-6533-0

\section{Personal Response}

Do you anticipate ANN neural networks will ever become $100 \%$ accurate at predicting the likelihood of pancrealic cancer?

II New pancreatic cancer biomarkers should be incorporated into a new version to improve the predictions made by PDAC-ANN. Although this could increase the performance, $100 \%$ accuracy is unlikely.
A 100\% accurate tool means that during the training phase, the key features and all possible variations were presented. As a consequence, nothing different from the training set is seen in new samples. If this is not the case, training a tool and reaching high values of is excellent in the training set but has low accuracy samples never seen before. Cancer development is a complex molecular disease that occurs after mutations and modifications in gene expression, and we are still finding all essential features and learning all variations. 